\title{
Historical remarks on equivalence of functions
}

by

\section{Zbigniew LIPECKI}

Summary. A theorem of E. Szpilrajn (Marczewski) (1936) on equivalent functions is recalled and its relation to subsequent results published by M. Morayne and C. RyllNardzewski (1999) and M. Kysiak (2005) is briefly discussed.

More than 80 years ago Edward Szpilrajn, who later changed his name to Marczewski, published a fundamental paper [8]. In that paper he defined the very useful notion of the characteristic function of a sequence of sets, nowadays often called the Marczewski function (see, e.g., [1, p. 9]). He used it to study the equivalence of sequences of sets, following a suggestion of Stanisław Ulam.

It is less known that in the same paper he also introduced and studied the notion of equivalence for functions (see $[8,2.1]$ ). To recall the corresponding definition, let $X, Y$ and $Z$ be sets and let $g: X \rightarrow Z$ and $h: Y \rightarrow Z$. The functions $g$ and $h$ are called equivalent if there exists a bijection $\varphi: X \rightarrow Y$ such that $g(x)=h(\varphi(x))$ for every $x \in X$. It is shown in [8, 2.4(i)] that $g$ and $h$ are equivalent if and only if $g^{-1}(z)$ and $h^{-1}(z)$ are equipotent for every $z \in Z$.

Let now $X$ be an uncountable Polish space and let $\boldsymbol{F}$ be a class of functions on $X$ with values in the unit interval $I$. Consider the following two conditions on $\boldsymbol{F}$ :

(a) For every $f \in \boldsymbol{F}$ there exists a perfect subset $P$ of $X$ such that $f \mid P$ is continuous.

(b) There is a subset $K$ of $X$ such that both $K$ and $X \backslash K$ have cardinality c and for every $f: X \rightarrow I$ we have $f \in \boldsymbol{F}$ whenever $f \mid K$ is Borel measurable.

2010 Mathematics Subject Classification: 28A05, 28A20, 26A99.

Key words and phrases: Polish space, equivalent functions, Lebesgue measurable function, function having the Baire property, Borel measurable function, function satisfying condition ( $s$, Marczewski measurable function.

Received 10 March 2017.

Published online 28 April 2017. 
As noted in [8, 3.5], these conditions are satisfied by the class of Lebesgue measurable functions (on $X=I^{n}$ ) and the class of functions having the Baire property (on $X=I^{n}$ ). The same is true for the class of functions satisfying condition $(s)$ (see [7] for definition), which are nowadays also called Marczewski measurable. The continuum hypothesis assumed in [8] in this last case turned out later to be superfluous (see 5. Chapter 5, Section I, Theorem 36]).

Let $U$ be a set of cardinality $\mathfrak{c}$. Then $f: U \rightarrow I$ is said to satisfy condition (A) if either $f(U)$ contains a perfect set or $f^{-1}\left(y_{0}\right)$ has cardinality $\mathfrak{c}$ for some $y_{0} \in I$.

With this notation, the following theorem holds (see [8, 3.4(iii)]):

Let $\boldsymbol{F}$ be a class of functions satisfying conditions (a) and (b) and let

$f: U \rightarrow I$. Then $f$ is equivalent to a function from $\boldsymbol{F}$ if and only if it satisfies condition (A).

More than 60 years later a special case of Marczewski's theorem, which applies to the class of Lebesgue measurable functions and that of functions having the Baire property, was rediscovered by Morayne and RyllNardzewski [4, Theorem]. That special case was then generalized by Kysiak [3, Theorem 5] to include the class of functions having property $(s)$. Kysiak's generalization is also a consequence of Marczewski's theorem. (Condition (a) above appears in [3] as the Weak Continuous Restriction Property.) Another related paper [2] does refer to [8], but mainly in connection with the definition of equivalent functions. In particular, the priority of [8] over [3] and [4] has been overlooked by the authors of [2].

In [3] an essential role is played by the class $\mathcal{H}(\mathcal{A})$ of sets that belong hereditarily to a given class $\mathcal{A}$ of sets. It is worth noting that, in another context, the same class already appeared in [6].

\section{References}

[1] K. P. S. Bhaskara Rao and B. V. Rao, Borel spaces, Dissertationes Math. (Rozprawy Mat.) 190 (1981), 62 pp.

[2] A. Komisarski, H. Michalewski and P. Milewski, Functions equivalent to Borel measurable ones, Bull. Polish Acad. Sci. Math. 58 (2010), 55-64.

[3] M. Kysiak, Some remarks on indicatrices of measurable functions, Bull. Polish Acad. Sci. Math. 53 (2005), 281-284.

[4] M. Morayne and C. Ryll-Nardzewski, Functions equivalent to Lebesgue measurable ones, Bull. Polish Acad. Sci. Math. 47 (1999), 263-265.

[5] J. C. Morgan II, Point Set Theory, Dekker, New York, 1990.

[6] E. Szpilrajn (Marczewski), Sur certains invariants de l'opération (A), Fund. Math. 21 (1933), 229-235; reprinted in: E. Marczewski, Collected Mathematical Papers, Inst. Math., Polish Acad. Sci., Warszawa, 1996, 50-56. 
[7] E. Szpilrajn (Marczewski), Sur une classe de fonctions de M. Sierpiński et la classe correspondante d'ensembles, Fund. Math. 24 (1935), 17-34; reprinted in: E. Marczewski, Collected Mathematical Papers, Inst. Math., Polish Acad. Sci., Warszawa, 1996, 92-109.

[8] E. Szpilrajn (Marczewski), Sur l'équivalence des suites d'ensembles et l'équivalence des fonctions, Fund. Math. 26 (1936), 302-326; Correction, Fund. Math. 27 (1936), 294; reprinted in: E. Marczewski, Collected Mathematical Papers, Inst. Math., Polish Acad. Sci., Warszawa, 1996, 124-148.

Zbigniew Lipecki

Institute of Mathematics

Polish Academy of Sciences, Wrocław Branch

Kopernika 18

51-617 Wrocław, Poland

E-mail: lipecki@impan.pan.wroc.pl 
